\title{
RESPONSE OF PULMONARY ARTERY PRESSURE AND TOTAL PULMONARY RESISTANCE OF UNTRAINED, CONVALES- CENT MAN TO PROLONGED MILD STEADY STATE EXERCISE ${ }^{1}$
}

\author{
By SALVATORE M. SANCETTA, AND LOUIS RAKITA WITH THE TECHNICAL \\ ASSISTANCE OF GLADYS HECKMAN AND HANNA JANOUSKOVEC
}

(From the Department of Medicine, Cleveland City Hospital, and Western Reserve University School of Medicine, Cleveland, O.)

(Submitted for publication January 17, 1957; accepted March 7, 1957)

Although many published studies relevant to the effects of exercise on the gross pulmonary hemodynamics of patients with heart disease are available in the literature, few data are to be found which deal with normal man $(1-6)$. There have been conflicting claims that there is no increase, no significant increase, a consistent increase, or an occasional decrease in the pulmonary artery pressure of normal man as the result of exercise loads eventuating in oxygen uptakes up to $400 \mathrm{ml}$. per M. ${ }^{2}$ per min. (2) and pulmonary blood flows two to three times the resting basal levels $(1,5)$. Moreover, comparison among the published data is difficult because of variations in the exercise posture, the duration of exercise, the work loads employed, and the timing of blood and pressure sampling, which often are varied in different subjects within the same body of data.

Slonim, Ravin, Balchum, and Dressler (6) alone have systematically studied the continuous pulmonary artery pressure variations in 5 normal subjects in the recumbent position over a 7-minute period of exercise, employing an almost uniform work rate between 784 and 840 foot-pounds per minute. These authors noted immediate increases in pulmonary systolic, diastolic and mean pressures, which usually reached a peak between the first and third minutes of exercise with an overall tendency to a slight secondary decline by the sixth minute. A steady state was not achieved.

The present report is intended to extend these findings employing a mild and uniform external work load of 850 foot-pounds per minute sustained over a period of 39 minutes. This mild amount of work was selected in order that steady state be attained in a reasonably uniform period of time,

\footnotetext{
1 Supported by a grant from United Appeal, Lorain County, Ohio.
}

that it be maintained as long as possible, and that some lower limit of valid, measurable change be established for comparison in subjects with heart disease physically unable to exercise at higher work loads and achieve the steady state (7).

\section{MATERIALS AND METHODS}

Studies were conducted on 12 subjects with normal cardio-respiratory systems who had convalesced from various illnesses (bronchopneumonia, active peptic ulcer, eczematoid dermatitis), or had been admitted for investigation of minor complaints. None were anemic or febrile. All had been ambulatory for several days before the studies were conducted. On the day prior to the experiment the subjects were exercised in the supine position at a standard work rate of 850 -foot pounds per minute for a period of 39 minutes. In all but three instances the steady state, as determined by repetitive determinations of the oxygen consumption (8), was attained within 10 to 12 minutes of exercise; in the remaining three, it was attained at the 12 to 14 -minute period. The following morning, at an ambient temperature of 23 degrees $\mathrm{C}$., cardiac catheterization was performed in the post-absorptive state with mild sedation (Pentobarbital Sodium, $0.1 \mathrm{Gm}$.), employing standard No. 7F Cournand catheters. The supine position was maintained throughout the entire study. Following initial determinations of the right atrial, ventricular, pulmonary arterial and wedge pressures, all of these being within normal limits (9), the catheter tip was uniformly positioned in the right pulmonary artery, just to the right of the sternum. An indwelling arterial needle was placed into the brachial artery for the collection of blood specimens and the recording of peripheral arterial pressures. The heart rate was measured electrocardiographically and the respiratory rate was counted by direct observation of the flutter valve in the face mask. After a period of stabilization, control records were made of the pulmonary and brachial artery pressures and the heart and respiratory rates at the beginning of each of 3 consecutive minutes. This was followed immediately by duplicate 2 -minute determinations of the oxygen consumption and cardiac output employing the Fick principle, and two more recordings of pressures, heart, and respiratory rates. These constituted 
the control data. The legs were then elevated, and the feet secured to the pedals of a variable resistance bicycle ergometer (10). The entire maneuver consumed 3 to 4 minutes. A record was then made of pulmonary and brachial artery pressures and heart and respiratory rates at the beginning of each minute for 5 minutes in this stationary position, followed by exercise at a steady work rate of 850 foot-pounds per minute. The stroke radius was $18 \mathrm{~cm}$., and the thighs did not exceed a maximal elevation of 80 degrees from the horizontal, minimizing the possibility of abdominal compression.

In 7 patients (Table IA) arterial pressures and heart and respiratory rates were recorded at the beginning of each consecutive minute of exercise for 39 minutes, except during the 10 to 14,20 to 24 , and 30 to 34 -minute periods, when duplicate determinations were made of the oxygen consumption and the cardiac output. In 3 patients (Table IB) the identical procedure was carried out, except that an intra-arterial needle was not introduced and no blood specimens were withdrawn, since it was possible that recorded pressure levels could be affected by the short-term removal of numerous blood samples. In a third group of 2 patients (Table II), following the same procedure as with the first 7 , the work load was suddenly doubled at the end of 29 minutes without interruption of exercise. Recordings of pressures and rates were made every minute until a total elapsed time of 49 minutes, except during the 40 to 44 -minute period when cardiac output determinations were done. At the end of this time pressures were obtained during withdrawal of the catheter in the main pulmonary artery and the right ventricle, and the experiments were abruptly terminated. The latter experiments were done to note if any change in the previously established response pattern of the pulmonary artery pressure would occur with a sudden increment in the work load.

Pressures were transduced via P23A Statham strain gauges and recorded on the Brush Multi-channel directwriting oscillograph. ${ }^{2}$ The reference point for central venous pressures was $10 \mathrm{~cm}$. anterior to the posterior surface of the thorax, in the fourth intercostal space. Mean pressures were derived by planimetric integration of the pressure pulses in two consecutive, technically acceptable respiratory cycles. Exhaled air was measured through a dry gas test meter, 2-minute collections were made in Douglas bags and the samples analyzed in the Pauling oxygen analyzer. All expressed values for minute ventilation and oxygen consumption were corrected for STPD. The recorded data for oxygen consumptions and cardiac outputs represent the averages of two consecutive determinations which were required to

\footnotetext{
2 The sensitivity employed for pressure recording was $1 \mathrm{~mm}$. deflection for $1 \mathrm{~mm}$. $\mathrm{Hg}$ in the case of the pulmonary, and $1 \mathrm{~mm}$. deflection for $6 \mathrm{~mm}$. $\mathrm{Hg}$ in the case of the brachial artery pressures. Error through amplifier drift was avoided by careful checking of the zero line before and introduction of a zero line after each set of recordings.
}

vary less than 10 per cent (8) from each other to be accepted as technically valid. Blood oxygen values were determined in duplicate spectrophotometrically (11) and were required to vary less than 0.2 volume per cent from each other. Total pulmonary and estimated systemic resistances were calculated according to standard formulae and expressed as c.g.s. units (2). Mean pulmonary artery pressure only was employed in the case of the former, and those data indicate total pulmonary and not pulmonary vascular resistance. In choosing the values of mean pressure to be employed in the numerator of the formula, the average of the two pressure determinations preceding and the three immediately following each set of oxygen consumptions was employed. This minimized the error inherent to the selection of a single pressure point.

\section{RESULTS}

The data for the first group of 7 subjects are presented in Table IA, those for the control subjects in Table IB, and those for the 2 patients who underwent more prolonged exercise with an increase in the work load in Table II. The pressure values in the tables represent the averages of the individual minute-to-minute pressures for the recumbent control and stationary leg-up positions, and the 1 to 9,15 to 19,25 to 29,35 to 39 -minute periods of exercise (Tables IA and IB), or 1 to 9 , 15 to 19,25 to 29,30 to 39 , and 45 to 49 -minute periods (Table II). Typical exercise response patterns are shown in Figures 1, 2, and 3. Average changes for pressure and other data in the first 7 patients, expressed as percentile changes from the control data, are illustrated in Figure 4.

A steady state of oxygen consumption and cardiac output was maintained at the end of the first and second sets of exercise determinations in 11 patients (oxygen uptake only in the control subjects), the lone exception being W.A. (Table IA), in whom there was a progressive rise in the cardiac output. In patients A.V., C.Q., B.B., and L.P. (Table IA) the steady state was maintained to the end of exercise, whereas in W.A., V.P., and I.R. there was an increase in the cardiac output during the third period of exercise. The first level of exercise steady state was deliberately lost in T.P. and M.G. (Table II) since the work load was doubled. Despite these changes in oxygen consumption and cardiac output the presented values are considered reasonably valid since consecutive duplicate determinations differed less than 10 percent from each other. The minute ventilation: and respiratory rates followed rather closely the 
TABLE IA

Prolonged mild exercise-Work load: 850 foot-pounds

\begin{tabular}{|c|c|c|c|c|c|c|c|c|c|c|c|c|c|c|c|c|c|c|}
\hline \multirow[b]{2}{*}{$\begin{array}{l}\text { Pt. } \\
\text { Age }\end{array}$} & \multirow[b]{2}{*}{$\begin{array}{l}\text { Sex } \\
\text { B.S. }\end{array}$} & \multicolumn{4}{|c|}{$\begin{array}{l}\text { Oxygen consumption } \\
\text { ml./M. } / \mathrm{min} \text {. }\end{array}$} & \multicolumn{4}{|c|}{$\begin{array}{l}\text { Cardiac index } \\
\text { L./M.2/min. }\end{array}$} & \multicolumn{4}{|c|}{$\begin{array}{c}\text { Minute ventilation } \\
L . / M .2 / m i n\end{array}$} & \multicolumn{5}{|c|}{ Respiratory rate } \\
\hline & & $\begin{array}{l}\text { Con- } \\
\text { trol }\end{array}$ & $\begin{array}{l}\text { 10-14 } \\
\text { min. }\end{array}$ & $\begin{array}{l}20-24 \\
\text { min. }\end{array}$ & $\begin{array}{l}\text { min. } \\
\text { min }\end{array}$ & Con- & $\begin{array}{l}\text { 10-14 } \\
\text { min. }\end{array}$ & $\begin{array}{l}20-24 \\
\text { min. }\end{array}$ & $\begin{array}{l}\text { 30-34 } \\
\text { min. }\end{array}$ & Con- & $\begin{array}{l}\text { 10-14 } \\
\text { min. }\end{array}$ & $\begin{array}{l}\text { 20-24 } \\
\text { min. }\end{array}$ & $\begin{array}{l}\text { 30-34 } \\
\text { min. }\end{array}$ & Con- & $\begin{array}{c}\text { Feet } \\
\text { up }\end{array}$ & $\begin{array}{l}\text { 10-14 } \\
\text { min. }\end{array}$ & $\begin{array}{l}20-24 \\
\text { min. }\end{array}$ & $\begin{array}{l}\text { 30-34 } \\
\text { min. }\end{array}$ \\
\hline A. V. & $\begin{array}{l}\text { WM } \\
1.95\end{array}$ & 142 & 231 & 233 & 234 & 4.03 & 5.05 & 4.93 & 4.87 & 3.86 & 4.82 & 5.24 & 5.40 & 13.6 & 13.8 & 16.6 & 16.7 & 17.2 \\
\hline w. $_{23} \mathrm{~A}$. & $\begin{array}{l}\text { BM } \\
\mathbf{1 . 8 2}\end{array}$ & 109 & 202 & 202 & 212 & 3.06 & 3.90 & 4.14 & 4.41 & 3.86 & 5.30 & 5.36 & 5.55 & 24.0 & 24.0 & 25.3 & 25.4 & 26.0 \\
\hline $\mathrm{V}_{\mathbf{3 4}} \mathbf{P}$ & $\begin{array}{l}\text { WM } \\
1.86\end{array}$ & 114 & 211 & 207 & 212 & $\quad 2.78$ & 4.13 & 4.06 & 4.52 & 3.45 & 6.05 & 5.82 & 6.10 & 16.0 & 18.0 & 22.2 & 23.2 & 23.6 \\
\hline c. 2. & $\underset{1.45}{B F}$ & 100 & 174 & 166 & 174 & 2.87 & 4.02 & 3.91 & 4.04 & 3.47 & 4.93 & 4.66 & 4.85 & 16.0 & 16.0 & 19.6 & 19.6 & 20.4 \\
\hline I. $\mathbf{R}$. & $\begin{array}{l}\text { WM } \\
1.64\end{array}$ & 123 & 196 & 199 & 210 & 3.19 & 3.82 & 3.80 & 4.16 & 3.39 & 4.31 & 4.56 & 4.87 & 14.0 & 15.2 & 19.8 & 19.6 & 19.6 \\
\hline B. $\mathbf{5 5}$ & $\begin{array}{l}\text { WM } \\
1.64\end{array}$ & 103 & 197 & 201 & 202 & 2.17 & 2.95 & 3.01 & 3.00 & 3.33 & 4.60 & 4.96 & 5.13 & 16.0 & 16.0 & 20.0 & 20.0 & 18.0 \\
\hline L. $\mathbf{P}$. & $\begin{array}{c}\mathrm{BM} \\
1.67\end{array}$ & 115 & 224 & 214 & 215 & 2.60 & 3.62 & 3.59 & 3.66 & 4.02 & 6.59 & 6.28 & 6.40 & 14.0 & 14.0 & 18.0 & 18.0 & 18.0 \\
\hline
\end{tabular}

* Key: W, white; B, black; M, male; B.S., body surface in square meters. Systolic, mean, and diastolic pressures from above downward in each pressure data group. The data for respiratory rate, pulmonary and brachial artery pressure represent the averages of the points sampled for each time period indicated. Blank spaces indicate technical inability to obtain data.

changes in oxygen consumption, remaining fairly stable at the end of the first two exercise periods, and tending to increase toward the end of exercise.

Elevation of the legs to the exercise position resulted in an increase in both pulmonary and brachial artery pressure in almost all instances. The pulmonary artery pressure presented two response patterns during exercise. In most cases, as exemplified in Figure 1, there was a rapid rise which remained variably sustained for the first 5 or 6 minutes. Thereafter there ensued a gradual decline so that by the end of the exercise period the pressures were only slightly above or occasionally below the control values. This was particularly true of the systolic and mean pressures. The diastolic pressure tended to show a lesser amount of decline. The second and less frequent response, as exemplified by Figure 2 (C.Q.), showed a more gradual and lesser rise in pressure which thereafter also declined progressively. This same pattern was noted in patients L.P. (IA) and J.G. (IB). In all three of these instances, the preponderant number of the pressure points recorded during exercise fell below the average and even below the lowest single pressure recorded in the control period. In no case, however, was there a failure to record at least several single pressures in the first 9 minutes which were not greater than the highest single value in the control period.

In the two subjects in whom the exercise load

TABLE II

Initial work load: 850 foot-pounds per minute-Work load increased to 1,700

\begin{tabular}{|c|c|c|c|c|c|c|c|c|c|c|c|c|c|c|c|c|c|c|}
\hline \multirow[b]{2}{*}{$\begin{array}{l}\text { Pt. } \\
\text { Age }\end{array}$} & \multirow[b]{2}{*}{$\begin{array}{l}\text { Sex } \\
\text { B.S. }\end{array}$} & \multicolumn{4}{|c|}{$\begin{array}{l}\text { Oxygen consumption } \\
m b . / M .2 / \min \text {. }\end{array}$} & \multicolumn{4}{|c|}{$\begin{array}{l}\text { Cardiac index } \\
L . / M .2 / \min .\end{array}$} & \multicolumn{4}{|c|}{$\begin{array}{c}\text { Minute ventilation } \\
L . / M .2 / \min .\end{array}$} & \multicolumn{5}{|c|}{ Respiratory rate } \\
\hline & & $\begin{array}{l}\text { Con- } \\
\text { trol }\end{array}$ & $\begin{array}{l}10-14 \\
\min .\end{array}$ & $\begin{array}{l}20-24 \\
\text { min. }\end{array}$ & $\begin{array}{l}40-44 \\
\text { min. }\end{array}$ & Con- & $\begin{array}{l}10-14 \\
\min .\end{array}$ & $\begin{array}{l}20-24 \\
\text { min. }\end{array}$ & $\begin{array}{l}40-44 \\
\min .\end{array}$ & Con- & $\begin{array}{l}10-14 \\
\min .\end{array}$ & $\begin{array}{l}20-24 \\
\min .\end{array}$ & $\begin{array}{l}\text { 40-44 } \\
\text { min. }\end{array}$ & $\begin{array}{c}\text { Con- } \\
\text { trol }\end{array}$ & $\begin{array}{c}\text { Feet } \\
\text { up }\end{array}$ & $\begin{array}{l}\text { 10-14 } \\
\text { min. }\end{array}$ & $\begin{array}{l}20-24 \\
\text { min. }\end{array}$ & $\begin{array}{l}40-44 \\
\text { min. }\end{array}$ \\
\hline T.P. & $\begin{array}{l}\text { BM } \\
1.72\end{array}$ & 109 & 201 & 206 & 280 & 2.13 & 3.44 & 3.46 & 4.03 & 3.11 & 5.63 & 5.75 & 6.85 & 12.0 & 12.0 & 16.5 & 16.6 & 20.7 \\
\hline $\mathbf{M .}_{32}$ G. & $\begin{array}{l}\text { BF } \\
1.90\end{array}$ & 84 & 191 & 192 & 287 & 2.30 & 3.40 & 3.48 & 4.00 & 2.60 & 4.92 & 5.13 & 6.68 & 12.0 & 12.0 & 16.4 & 16.8 & 17.6 \\
\hline
\end{tabular}

- Kog: W - Wite; B black; $M$ male; B.8, body gurface in square meters. Systolic, mean, and diastolic pressures from above downward in each preesure date group. The data for respiratory rate, pulmonary and brachial artery pressure represent the averages of the points sampled for each time period indicated. 
per minute-Total exercise time: 39 minutes*

TABLE IA-Continued

\begin{tabular}{|c|c|c|c|c|c|c|c|c|c|c|c|c|c|c|c|c|c|c|c|}
\hline \multicolumn{6}{|c|}{$\begin{array}{l}\text { Pulmonary arterial pressure } \\
\mathrm{mm} . \mathrm{Hg}\end{array}$} & \multicolumn{6}{|c|}{$\begin{array}{c}\text { Brachial arterial pressure } \\
\mathbf{m m} . \mathbf{H g}\end{array}$} & \multicolumn{4}{|c|}{$\begin{array}{l}\text { Total pulmonary } \\
\text { registance } \\
\text { C.G.S. units }\end{array}$} & \multicolumn{4}{|c|}{$\begin{array}{l}\text { Total peripheral } \\
\text { resistance } \\
\text { C.G.S. wnits }\end{array}$} \\
\hline $\begin{array}{c}\text { Con- } \\
\text { trol }\end{array}$ & $\begin{array}{l}\text { Feet } \\
\text { up }\end{array}$ & $\begin{array}{l}1-9 \\
\text { min. }\end{array}$ & $\begin{array}{l}15-19 \\
\text { min. }\end{array}$ & $\begin{array}{l}25-29 \\
\min .\end{array}$ & $\begin{array}{l}35-39 \\
\text { min. }\end{array}$ & $\begin{array}{l}\text { Con- } \\
\text { trol }\end{array}$ & $\begin{array}{c}\text { Feet } \\
\text { up }\end{array}$ & $\underset{\min }{1-9}$ & $\begin{array}{l}\text { 15-19 } \\
\text { min. }\end{array}$ & $\begin{array}{l}25-29 \\
\text { min. }\end{array}$ & $\begin{array}{l}\text { 35-39 } \\
\text { min. }\end{array}$ & $\begin{array}{l}\text { Con- } \\
\text { trol }\end{array}$ & $\begin{array}{l}10-14 \\
\min .\end{array}$ & $\begin{array}{l}20-24 \\
\text { min. }\end{array}$ & $\begin{array}{l}30-34 \\
\min \end{array}$ & $\begin{array}{l}\text { Con- } \\
\text { trol }\end{array}$ & $\begin{array}{l}10-14 \\
\text { min. }\end{array}$ & $\begin{array}{l}20-24 \\
\text { min. }\end{array}$ & $\begin{array}{l}30-34 \\
\text { min. }\end{array}$ \\
\hline $\begin{array}{r}22.2 \\
14.0 \\
9.5\end{array}$ & $\begin{array}{l}25.1 \\
16.0 \\
10.6\end{array}$ & $\begin{array}{l}25.9 \\
17.3 \\
10.7\end{array}$ & $\begin{array}{r}22.3 \\
15.1 \\
9.5\end{array}$ & $\begin{array}{r}21.4 \\
14.1 \\
9.5\end{array}$ & $\begin{array}{r}21.1 \\
14.0 \\
9.3\end{array}$ & $\begin{array}{r}111.0 \\
73.2 \\
55.0\end{array}$ & $\begin{array}{r}119.9 \\
80.9 \\
62.2\end{array}$ & $\begin{array}{r}126.5 \\
85.9 \\
67.7\end{array}$ & $\begin{array}{r}122.6 \\
83.0 \\
65.2\end{array}$ & $\begin{array}{r}126.4 \\
81.0 \\
69.6\end{array}$ & $\begin{array}{r}120.0 \\
84.0 \\
70.0\end{array}$ & 142 & 133 & 118 & 105 & 743 & 695 & 687 & 698 \\
\hline $\begin{array}{l}15.7 \\
12.4 \\
9.3\end{array}$ & $\begin{array}{l}19.0 \\
14.3 \\
11.1\end{array}$ & $\begin{array}{l}21.3 \\
16.4 \\
12.5\end{array}$ & $\begin{array}{l}19.6 \\
15.6 \\
12.3\end{array}$ & $\begin{array}{l}19.1 \\
15.4 \\
12.6\end{array}$ & $\begin{array}{l}17.5 \\
15.2 \\
11.5\end{array}$ & $\begin{array}{r}121.5 \\
85.0 \\
71.0\end{array}$ & $\begin{array}{r}132.2 \\
92.6 \\
78.0\end{array}$ & $\begin{array}{r}131.7 \\
87.1 \\
72.5\end{array}$ & $\begin{array}{r}137.8 \\
93.4 \\
78.0\end{array}$ & $\begin{array}{r}141.2 \\
99.9 \\
81.8\end{array}$ & $\begin{array}{r}138.8 \\
96.4 \\
78.4\end{array}$ & 178 & 175 & 164 & 155 & 1,217 & 1,022 & 1,018 & 980 \\
\hline $\begin{array}{r}18.9 \\
12.8 \\
9.0\end{array}$ & $\begin{array}{r}19.8 \\
14.4 \\
9.9\end{array}$ & $\begin{array}{l}22.9 \\
17.5 \\
13.5\end{array}$ & $\begin{array}{l}19.3 \\
15.1 \\
10.8\end{array}$ & $\begin{array}{l}18.3 \\
13.9 \\
10.4\end{array}$ & $\begin{array}{l}16.6 \\
12.9 \\
10.3\end{array}$ & $\begin{array}{l}95.8 \\
68.8 \\
53.2\end{array}$ & $\begin{array}{r}106.2 \\
76.8 \\
59.2\end{array}$ & $\begin{array}{l}99.7 \\
69.8 \\
53.5\end{array}$ & $\begin{array}{l}95.7 \\
66.9 \\
48.8\end{array}$ & $\begin{array}{l}98.8 \\
68.2 \\
51.4\end{array}$ & $\begin{array}{r}100.0 \\
68.5 \\
52.2\end{array}$ & 198 & 164 & 151 & 126 & 1,064 & 729 & 699 & 662 \\
\hline $\begin{array}{r}14.6 \\
9.6 \\
4.8\end{array}$ & $\begin{array}{r}15.9 \\
10.4 \\
5.9\end{array}$ & $\begin{array}{r}14.9 \\
10.0 \\
5.5\end{array}$ & $\begin{array}{r}14.0 \\
8.6 \\
4.7\end{array}$ & $\begin{array}{r}12.2 \\
8.0 \\
4.2\end{array}$ & $\begin{array}{r}12.5 \\
7.8 \\
3.8\end{array}$ & & & & & & & 184 & 131 & 110 & 110 & & & & \\
\hline $\begin{array}{r}24.4 \\
16.1 \\
8.4\end{array}$ & $\begin{array}{r}25.1 \\
16.0 \\
9.8\end{array}$ & $\begin{array}{l}28.7 \\
17.8 \\
11.4\end{array}$ & $\begin{array}{l}29.1 \\
17.8 \\
10.5\end{array}$ & $\begin{array}{r}27.5 \\
16.8 \\
9.7\end{array}$ & $\begin{array}{r}26.3 \\
16.4 \\
9.6\end{array}$ & & & & & & & 246 & 218 & 220 & 198 & & & & \\
\hline $\begin{array}{r}18.5 \\
13.2 \\
8.9\end{array}$ & $\begin{array}{r}20.4 \\
13.8 \\
9.4\end{array}$ & $\begin{array}{l}23.9 \\
17.3 \\
13.0\end{array}$ & $\begin{array}{l}23.1 \\
16.4 \\
12.1\end{array}$ & $\begin{array}{l}22.3 \\
15.8 \\
11.3\end{array}$ & $\begin{array}{l}21.8 \\
15.4 \\
10.5\end{array}$ & $\begin{array}{r}106.8 \\
81.4 \\
60.7\end{array}$ & $\begin{array}{r}123.1 \\
89.6 \\
67.8\end{array}$ & $\begin{array}{r}124.0 \\
88.6 \\
68.8\end{array}$ & $\begin{array}{r}124.1 \\
90.5 \\
67.6\end{array}$ & $\begin{array}{r}127.8 \\
94.1 \\
69.8\end{array}$ & $\begin{array}{r}118.2 \\
82.6 \\
61.2\end{array}$ & 296 & 274 & 259 & 253 & 1,828 & 1,467 & 1,501 & 1,412 \\
\hline $\begin{array}{r}18.4 \\
9.8 \\
5.4\end{array}$ & $\begin{array}{r}18.1 \\
10.4 \\
6.7\end{array}$ & $\begin{array}{r}16.9 \\
10.1 \\
7.1\end{array}$ & $\begin{array}{r}14.0 \\
9.0 \\
5.6\end{array}$ & $\begin{array}{r}13.4 \\
8.4 \\
5.1\end{array}$ & $\begin{array}{r}12.8 \\
7.5 \\
5.1\end{array}$ & $\begin{array}{r}138.7 \\
101.9 \\
83.8\end{array}$ & $\begin{array}{r}141.8 \\
103.1 \\
83.9\end{array}$ & $\begin{array}{r}137.4 \\
97.1 \\
79.6\end{array}$ & $\begin{array}{r}132.7 \\
93.6 \\
77.6\end{array}$ & $\begin{array}{r}132.6 \\
92.7 \\
78.5\end{array}$ & $\begin{array}{r}134.5 \\
91.3 \\
79.7\end{array}$ & 180 & 133 & 120 & 110 & 1,872 & 1,284 & 1,247 & 1,203 \\
\hline
\end{tabular}

TABLE IB

Control group-Brachial artery pressures and cardiac outputs not obtained *

\begin{tabular}{|c|c|c|c|c|c|c|c|c|c|c|c|c|c|c|c|}
\hline \multirow[b]{2}{*}{$\begin{array}{l}\text { Pt. } \\
\text { Age }\end{array}$} & \multirow[b]{2}{*}{ Sex } & \multicolumn{4}{|c|}{$\begin{array}{l}\text { Oxygen consumption } \\
\text { mi./M.2/min. }\end{array}$} & \multicolumn{4}{|c|}{$\begin{array}{c}\text { Minute ventilation } \\
\text { L. } / M .2 / \min \text {. }\end{array}$} & \multicolumn{6}{|c|}{$\begin{array}{l}\text { Pulmonary arterial pressure } \\
\text { mm. } \boldsymbol{H}_{\boldsymbol{g}}\end{array}$} \\
\hline & & $\begin{array}{l}\text { Con- } \\
\text { trol }\end{array}$ & $\begin{array}{l}10-14 \\
\text { min. }\end{array}$ & $\begin{array}{l}20-24 \\
\text { min. }\end{array}$ & $\begin{array}{l}\text { 30-34 } \\
\text { min. }\end{array}$ & Con- & $\begin{array}{l}\text { 10-14 } \\
\text { min. }\end{array}$ & $\begin{array}{l}20-24 \\
\text { min. }\end{array}$ & $\begin{array}{l}\text { 30-34 } \\
\text { min. }\end{array}$ & Con- & $\begin{array}{c}\text { Feet } \\
\text { up }\end{array}$ & $\begin{array}{c}1-9 \\
\text { min. }\end{array}$ & $\begin{array}{l}15-19 \\
\text { min. }\end{array}$ & $\begin{array}{l}25-29 \\
\text { min. }\end{array}$ & $\begin{array}{l}35-39 \\
\text { min. }\end{array}$ \\
\hline $\mathbf{J}_{23} \mathbf{G}$. & $\begin{array}{l}\text { WM } \\
1.80\end{array}$ & 110 & 193 & 195 & 186 & 3.07 & 4.92 & 5.14 & 5.31 & $\begin{array}{r}13.1 \\
8.7 \\
5.4\end{array}$ & $\begin{array}{r}13.5 \\
9.8 \\
6.9\end{array}$ & $\begin{array}{r}12.8 \\
10.1 \\
7.8\end{array}$ & $\begin{array}{r}13.8 \\
9.8 \\
7.1\end{array}$ & $\begin{array}{r}12.8 \\
8.7 \\
7.3\end{array}$ & $\begin{array}{r}12.1 \\
8.6 \\
6.8\end{array}$ \\
\hline L. $Q$. & $\begin{array}{l}\text { BF } \\
1.34\end{array}$ & 110 & 183 & 180 & 179 & 4.47 & 4.92 & 6.87 & 5.31 & $\begin{array}{r}11.2 \\
5.2 \\
2.8\end{array}$ & $\begin{array}{r}14.7 \\
6.7 \\
4.3\end{array}$ & $\begin{array}{r}17.2 \\
9.2 \\
4.8\end{array}$ & $\begin{array}{r}18.1 \\
8.7 \\
4.4\end{array}$ & $\begin{array}{r}16.5 \\
8.5 \\
3.7\end{array}$ & $\begin{array}{r}16.5 \\
7.9 \\
2.8\end{array}$ \\
\hline C. 58 & $\begin{array}{l}\text { WM } \\
1.58\end{array}$ & 107 & 217 & 214 & 216 & 4.06 & 6.91 & 6.78 & 6.83 & $\begin{array}{l}26.3 \\
14.6 \\
11.7\end{array}$ & $\begin{array}{l}27.5 \\
15.7 \\
13.6\end{array}$ & $\begin{array}{l}30.0 \\
19.0 \\
14.8\end{array}$ & $\begin{array}{l}26.2 \\
17.3 \\
14.1\end{array}$ & $\begin{array}{l}23.3 \\
14.7 \\
11.6\end{array}$ & \\
\hline
\end{tabular}

* Key: W, white; B, black; M, male; B.S., body surface in square meters. Systolic, mean, and diastolic pressures from above downward in each pressure data group. The data for respiratory rate, pulmonary and brachial artery pressure represent the averages of the points sampled for each time period indicated.

TABLE II-Continued

foot-pounds at 29.5 minutes of exercise-Total exercise time: 49 minutes *

\begin{tabular}{|c|c|c|c|c|c|c|c|c|c|c|c|c|c|c|c|c|c|c|c|c|c|}
\hline \multicolumn{7}{|c|}{$\begin{array}{l}\text { Pulmonary arterial presesuro } \\
\text { min. } H g\end{array}$} & \multicolumn{7}{|c|}{$\underset{\text { Brachial arterial presoure }}{\boldsymbol{H}_{\theta}}$} & \multicolumn{4}{|c|}{$\begin{array}{c}\text { Total polmonery } \\
\text { reagtance } \\
\text { C.GS. with }\end{array}$} & \multicolumn{4}{|c|}{$\begin{array}{c}\text { Total peripheral } \\
\text { regiatance } \\
\text { C.GS. wnits }\end{array}$} \\
\hline Con- & Feet & $\begin{array}{l}1-9 \\
\min \end{array}$ & $\begin{array}{l}15-19 \\
\min .\end{array}$ & $\begin{array}{l}25-20 \\
\min .\end{array}$ & $\begin{array}{l}30-39 \\
\min .\end{array}$ & $\begin{array}{l}45-19 \\
\min .\end{array}$ & Con- & $\underset{\text { up }}{\text { Feet }}$ & $\underset{\min }{1-0}$ & $\begin{array}{l}\text { 15-19 } \\
\min .\end{array}$ & $\begin{array}{l}20-29 \\
\min .\end{array}$ & $\begin{array}{l}30-00 \\
\text { min. }\end{array}$ & $\begin{array}{l}40-10 \\
\min .\end{array}$ & Con- & $\begin{array}{l}10-11 \\
\min .\end{array}$ & $\begin{array}{l}20-24 \\
\min .\end{array}$ & $\begin{array}{l}\frac{40-44}{\min .} \\
\text {. }\end{array}$ & Coon- & $\begin{array}{l}10-14 \\
\min .\end{array}$ & $\begin{array}{l}20-24 \\
\min .\end{array}$ & $\begin{array}{l}40-44 \\
\min .\end{array}$ \\
\hline $\begin{array}{r}18.5 \\
10.6 \\
7.7\end{array}$ & $\begin{array}{r}21.3 \\
12.9 \\
8.6\end{array}$ & $\begin{array}{l}21.2 \\
14.6 \\
10.4\end{array}$ & $\begin{array}{r}22.9 \\
12.8 \\
8.1\end{array}$ & $\begin{array}{r}21.1 \\
12.5 \\
8.3\end{array}$ & $\begin{array}{r}22.6 \\
13.8 \\
9.2\end{array}$ & $\begin{array}{r}18.7 \\
11.4 \\
7.4\end{array}$ & $\begin{array}{r}107.4 \\
80.8 \\
72.1\end{array}$ & $\begin{array}{r}107.8 \\
83.6 \\
71.2\end{array}$ & $\begin{array}{r}118.0 \\
87.8 \\
77.3\end{array}$ & $\begin{array}{r}115.4 \\
88.3 \\
74.8\end{array}$ & $\begin{array}{r}114.8 \\
83.4 \\
78.8\end{array}$ & $\begin{array}{r}122.6 \\
90.1 \\
78.3\end{array}$ & $\begin{array}{r}120.2 \\
88.3 \\
77.4\end{array}$ & 231 & 180 & 169 & 148 & 1,764 & 1,193 & 1,135 & 1,043 \\
\hline $\begin{array}{r}17.7 \\
13.2 \\
7.5\end{array}$ & $\begin{array}{r}21.8 \\
16.1 \\
8.4\end{array}$ & $\begin{array}{l}20.9 \\
17.2 \\
10.5\end{array}$ & $\begin{array}{r}18.1 \\
14.8 \\
8.3\end{array}$ & $\begin{array}{r}15.5 \\
13.2 \\
8.2\end{array}$ & $\begin{array}{r}17.9 \\
14.2 \\
9.0\end{array}$ & $\begin{array}{r}15.3 \\
12.0 \\
7.2\end{array}$ & $\begin{array}{r}111.1 \\
82.2 \\
62.7\end{array}$ & $\begin{array}{r}116.6 \\
88.2 \\
70.0\end{array}$ & $\begin{array}{r}119.1 \\
00.7 \\
70.0\end{array}$ & $\begin{array}{r}114.6 \\
85.4 \\
66.7\end{array}$ & $\begin{array}{r}111.7 \\
8.55 \\
64.0\end{array}$ & $\begin{array}{r}115.4 \\
8.4 .2 \\
66.6\end{array}$ & $\begin{array}{r}111.3 \\
79.1 \\
65.2\end{array}$ & 241 & 188 & 164 & 127 & 1,804 & 1,085 & 907 & 857 \\
\hline
\end{tabular}




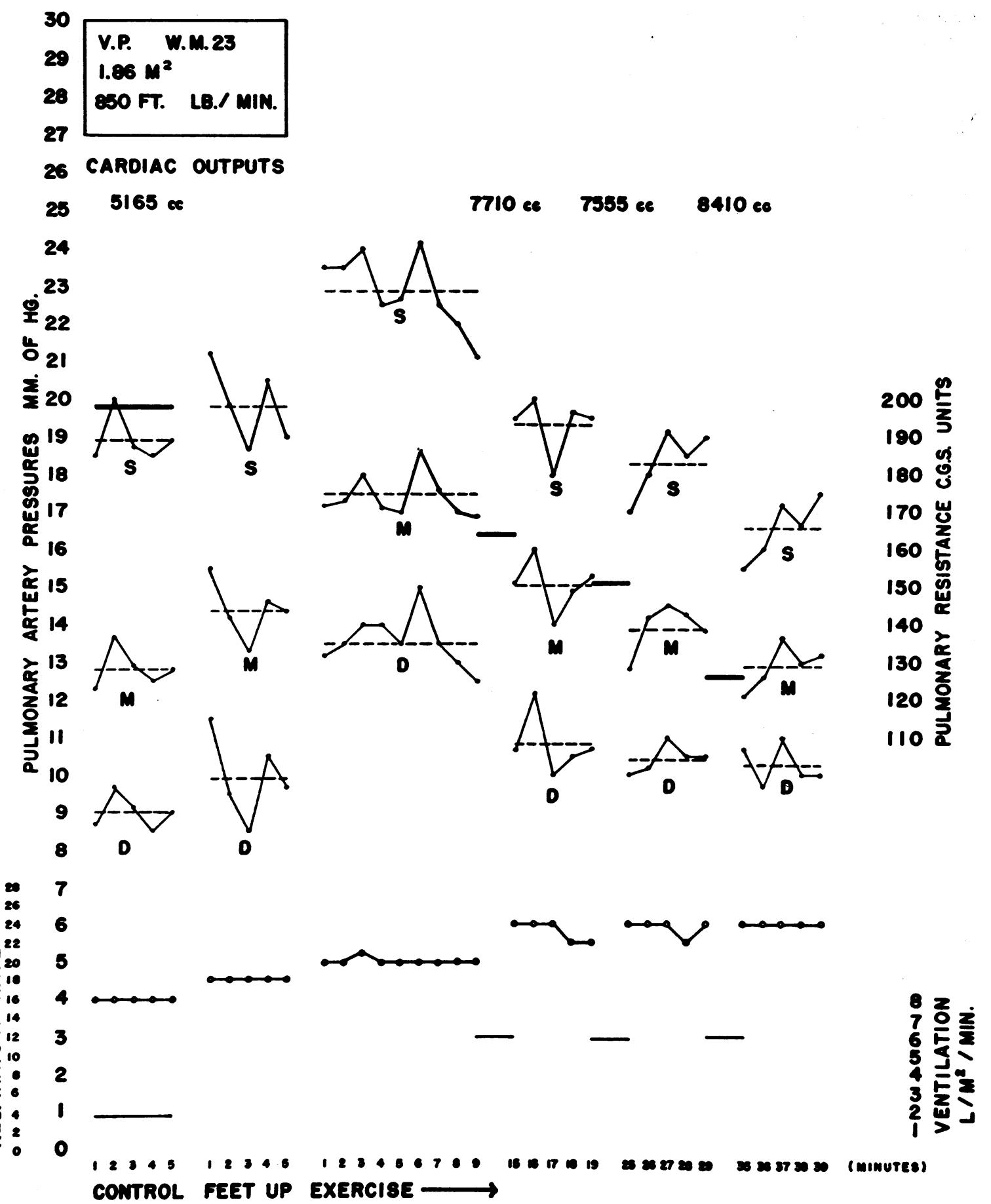

Fig. 1. Response of Pulmonary Artery Pressures, total Pulmonary Resistanct (Heavy Lines), Respiratory Rate (Circtes) and Ventilation in Patient V.P. (Seg Text)

$\mathrm{S}, \mathrm{M}$, and $\mathrm{D}=$ systolic, mean and diastolic pressures. The horizontal interrupted lines indicate the average value for each set of pressure points. 


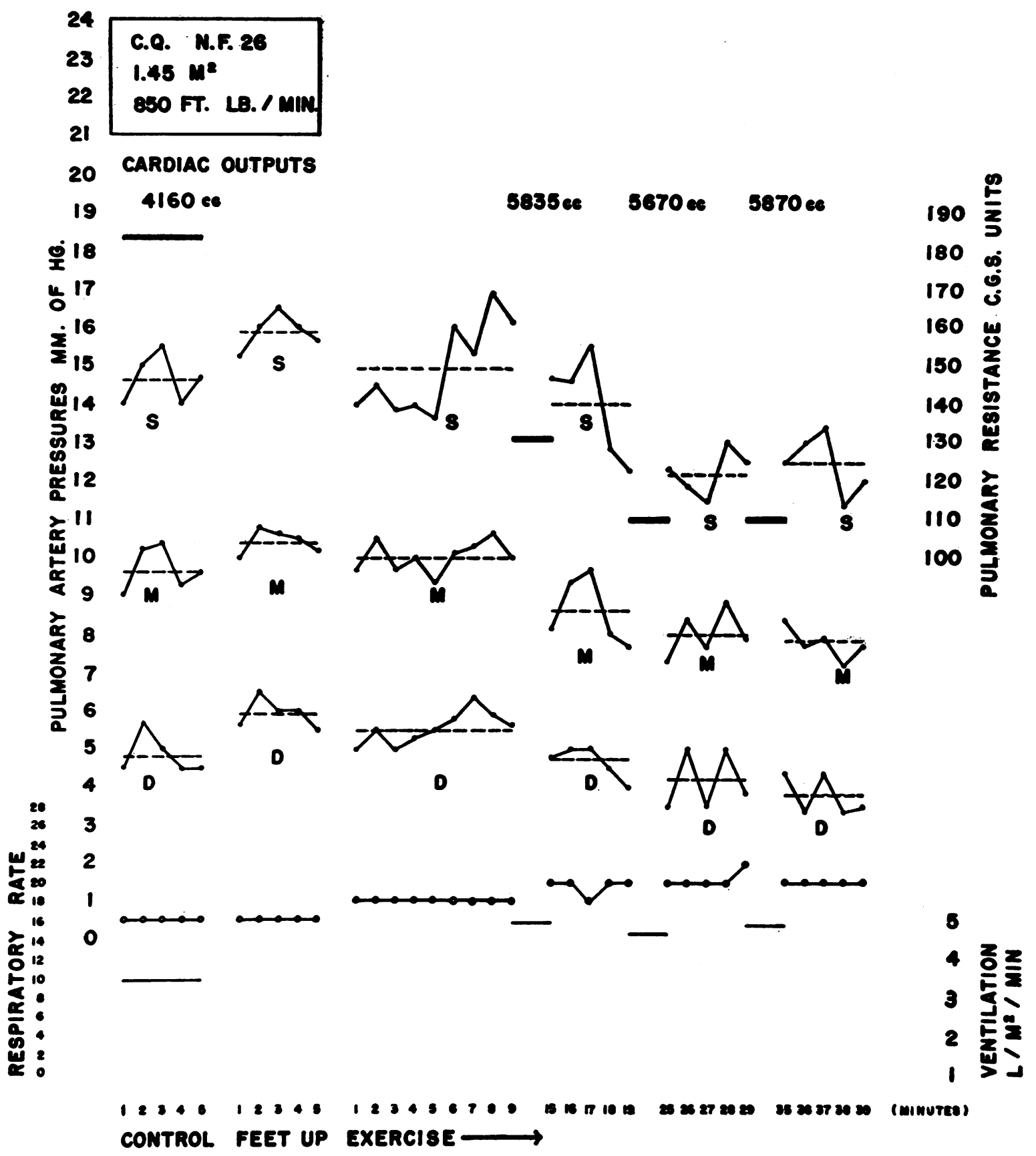

Fig. 2. Response of Pulmonary Artery Pressures, total Pulmonary Resistance (Heavy Lines), Respiratory Rate (Circles) and Ventilation in Patient C.Q. (See Text)

was doubled at the end of 29 minutes of exercise, as exemplified by patient T.P. (Figure 3 ), there was a secondary rise in pressure which did not, however, attain the same elevation as the rises recorded in the first 9 minutes of exercise. There- after, the pressures rapidly declined, and by the termination of exercise they were even lower than immediately prior to the increased work load.

The brachial artery pressure did not show the same repetitiveness of response to exercise as did 


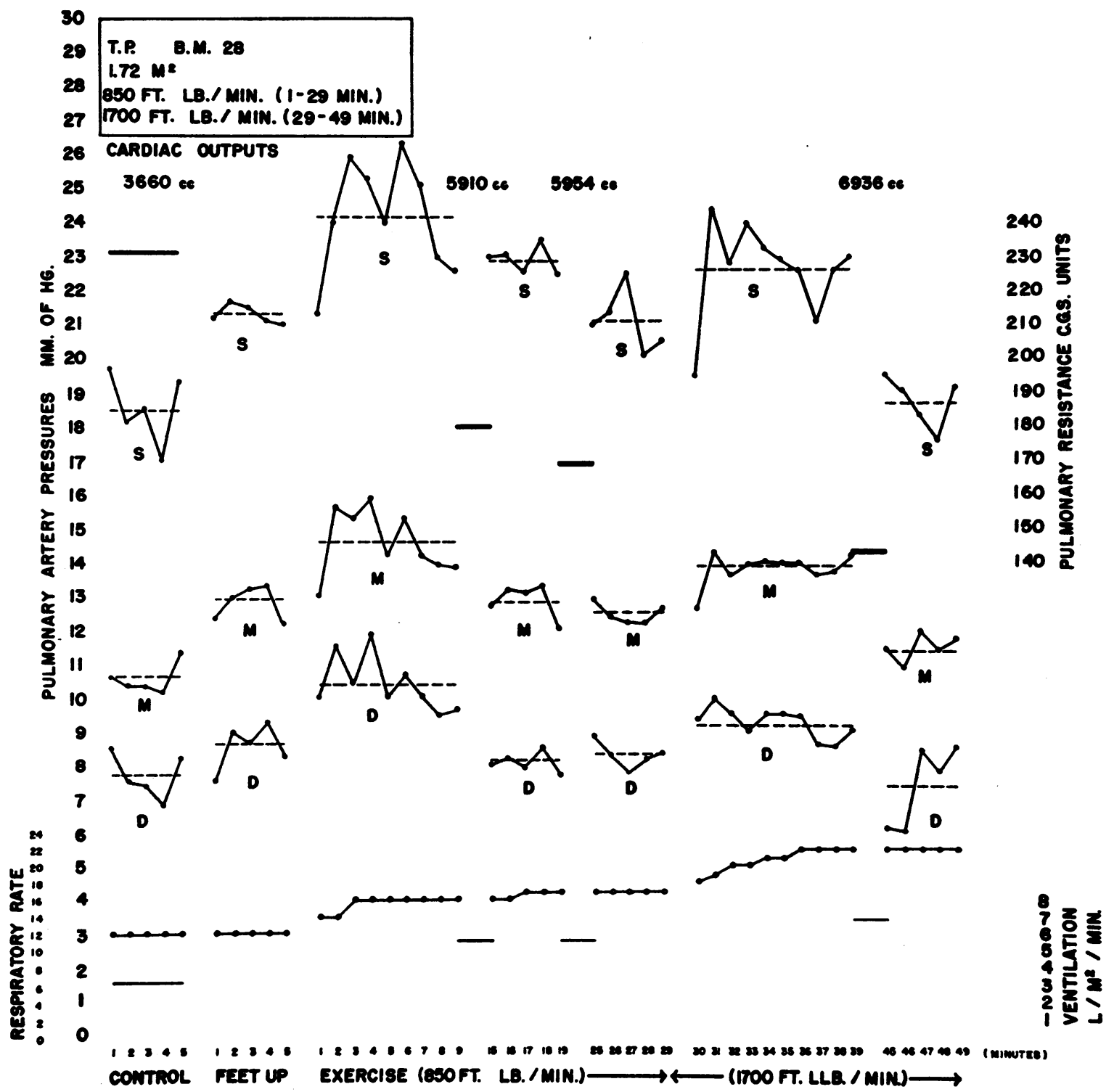

Fig. 3. Response of Pulmonary Artery Pressures, Total Pulmonary Resistance (Heavy Lines), Respiratory Rate (Circles) and Ventilation in Patient T.P.

The work load was doubled at 29.5 minutes of exercise (see text).

the pulmonary artery pressures, and on the average the values during the first 9 minutes of exercise were less than those recorded in the stationary leg-up position, though higher than in the control period. Throughout the entire period of exercise the pressures tended to deviate but little from the slight elevations recorded in the period of stationary leg elevation. There was no particular correlation either on the whole or in the individual subjects between the pulmonary and brachial artery pressure changes.

The total pulmonary resistance showed a progressive decrease from the control values throughout the entire exercise period, except for patients I.R. and C.Q. (Table IA), in whom there was no difference between the values at the end of the first and second, and the second and third periods of exercise, respectively. Otherwise, the progres- 
sive decreases in resistance occurred regardless of stability or change in the cardiac output.

The estimated total systemic resistance, on the other hand, showed some tendency to vary with the change in cardiac output, and on the average remained essentially unchanged when the latter remained steady, and decreased further when the output increased (Figure 4).

Although recorded, the data for the heart rate have not been presented, since they showed a rapid increase with exercise and a maintenance of a study plateau throughout the entire exercise period.

\section{DISCUSSION}

It must be noted at the outset that the changes in pulmonary resistance recorded in these experiments relate to the total circuit from the pulmonary artery to the left atrium. In the absence of data for the left atrial pressure, the entire circuit must be considered as a whole. It is not possible to dissociate alterations of resistance in the pulmonary vascular bed from those changes taking place within the heart chambers.

Although the pulmonary artery pressure changes reported in these experiments were small, they were nonetheless sequentially consistent, particularly when presented in terms of both individual and cumulative averages. These changes resemble generally the data of Slonim, Ravin, Balchum, and Dressler (6) for the first few minutes of exercise, in that a variable but consistent pressure increase was noted. The contribution to this pressure increase by passive elevation of the legs to the exercise position, however, appears to have been ignored in the published literature. Elevations in both pulmonary and brachial artery pressures were recorded in almost all of the experiments when the legs were raised. While the mechanism of these elevations has not been definitely elucidated, similar increases, incompletely reversed by ganglion blocking doses of hexamethonium, occur with the assumption of a 35-degree total head-down body tilt (12). It is possible that the recorded pressure increases in the pulmonary artery may be due to a translocation of blood from the lower extremities with a resultant increase in the intrathoracic blood volume (13). In any event, a portion of the pressure elevation occurring during recumbent exer- cise must be ascribed solely to passive elevation of the legs when a "bicycle" type of ergometer is employed.

As was noted earlier, the data reported by Slonim, Ravin, Balchum, and Dressler (6) seemed to indicate that following the initial rise in pulmonary artery pressure, there appeared some tendency to a slight secondary decrease by the sixth or seventh minutes of exercise, when the experiments were terminated. The present studies extend these findings and indeed demonstrate a gradual and continued decrease in pressure throughout the entire prolonged period of exercise. This same pattern was reproducible regardless of the degree of rise during the first few minutes of exercise, and was not due to the withdrawal of blood samples for analysis (Table IB), nor, as shown in previous studies employing a similar amount of exercise $(7,14)$, to any marked change in peripheral arterial $\mathrm{pCO}_{2}$ or oxygen saturation. It is possible that the initial pressure rise during the first few minutes of exercise may be caused by a further transient increase in the intrathoracic blood volume consonant with an increasing venous return. This possibility, although unsubstantiated, is rather attractive, and would explain nicely the temporary interruption of the progressive pressure decline noted in the two subjects in whom the work effort was doubled at the end of 29 minutes of exercise (Table II, Figure 3).

The compensatory readjustments producing the progressive pressure decrease after an initial rise may, however, be so manifold as to defy any single explanation of these findings. One may speculate that all or some of the following factors may play a part:

1. Verzár (15) has shown that factors which increase the respiratory effort in humans, such as exercise or exposure to low barometric pressures and to reduced concentrations of oxygen in the inspired air (10 and 7 per cent), produce an increase of the effective residual pulmonary volume. Although there is no incontrovertible proof (15), this might be expected to produce an increased negativity of the intrapleural pressure and a consequent decrease in the intravascular pressure. This would not appear to be the sole factor involved, however, since respiratory effort ap- 


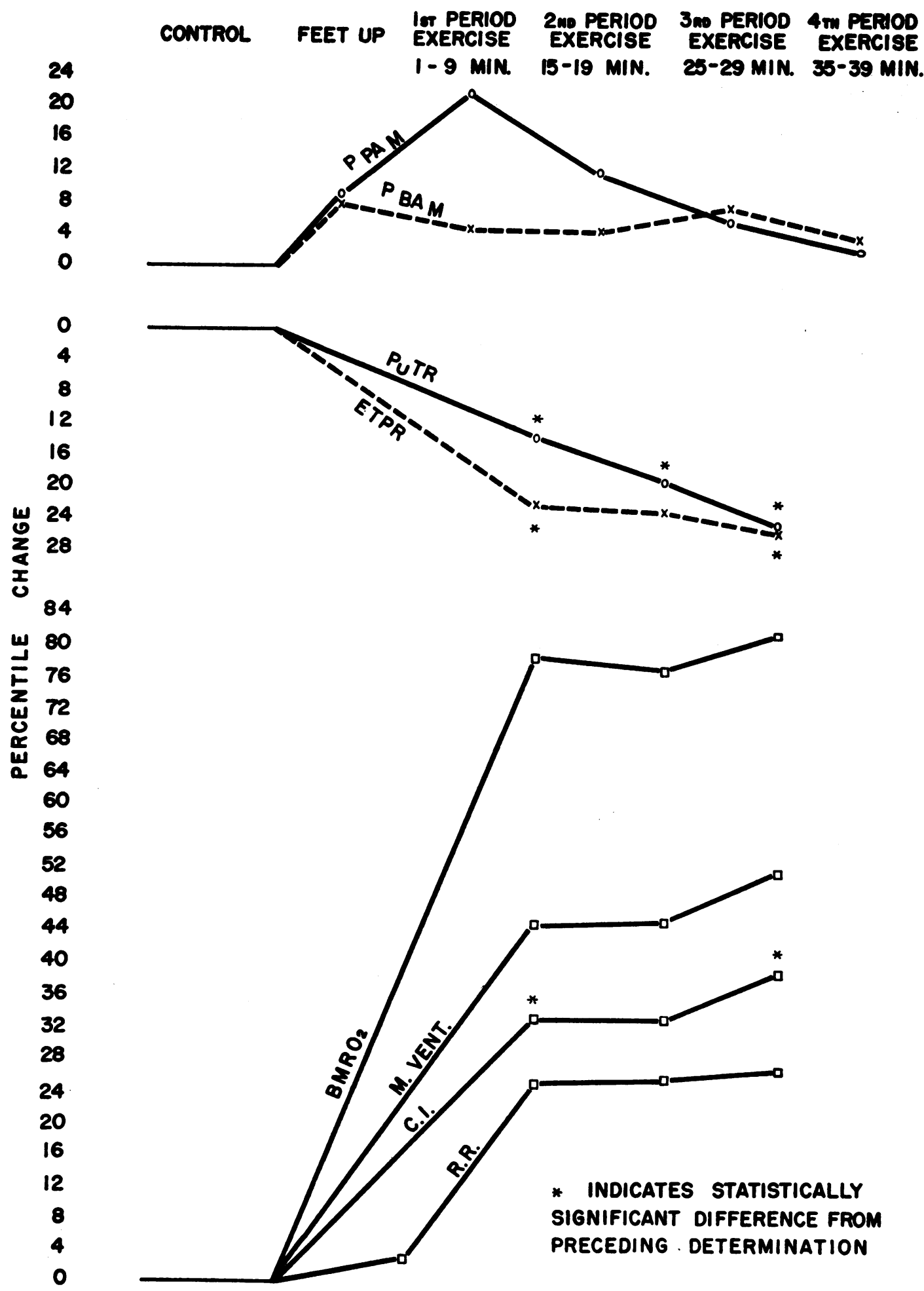

Fig. 4. Average Percentile Change from Control Data for Pulmonary (Ppam) and Brachial

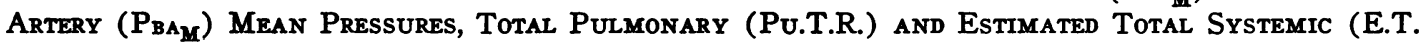
P.R.) Resistances, Oxygen Consumption (BMRO $)_{2}$, Minute Ventilation, Cardiac Index (C.I.) and Respiratory Rates (R.R.) for the 7 Patients in Table I

Note that there is no significant difference between E.T.P.R. following the first and second exercise periods and the cardiac index for the same periods, indicating maintenance of exercise steady state for these two modalities, and an absence of steady state of Pu.T.R. (Fisher's t test, groups less than 30) throughout the entire exercise period. There is loss of the steady state for the cardiac index and the E.T.P.R. following the third period of exercise. The other data were not analyzed statistically. 
peared essentially unchanged following the first and second periods of exercise, as gauged roughly by the respiratory and ventilatory rates.

2. There seems to be good evidence that in the resting basal state not all pulmonary capillaries are functional at any given moment (16). A fair amount of evidence has also accumulated that pulmonary arteriovenous anastomoses exist in mammalian (16-19) lungs. Lilienthal and Riley (16) opine that such anastomoses are not functional in the resting state, but that a relatively small stimulus may cause them to open. If this be true, and an initial increase in intrathoracic blood volume is gradually reversed (the anastomoses and capillaries meantime remaining patent, Reference $19^{3}$ ), pulmonary artery pressure would gradually decrease although a steady state of cardiac output is maintained. It is evident from the data herein presented that a steady state of cardiac output was maintained at the end of the first and second exercise periods in 8 of 9 experiments.

3. As a result of the increased pulmonary blood flow there may occur increased pressure distention of the pulmonary vascular bed (20); this may conceivably be followed by a progressive decrease in intrinsic or neurogenically controlled vascular "tone" as a compensatory phenomenon.

4. Finally, it is not possible to assess the impression of peripheral arterial changes upon the pulmonary artery pressure, in view of the contradictory evidence relevant to the existence of pulmonary-bronchial artery anastomoses in normal human lungs $(21,22)$. While the absence of correlation between the peripheral and pulmonary arterial pressure changes in the present data (Figure 4) confirms the original observations of Bradford and Dean (23), there is no assurance that an interplay of forces may not occur.

Regardless of what compensatory mechanisms lead to this adaptation of the cardiopulmonary bed to an increased blood flow, it is noteworthy that total pulmonary resistance decreased progressively throughout the exercise period. While this would

\footnotetext{
${ }^{3}$ Rahn, Stroud, and Tobin showed by injection of thorotrast through a catheter lodged into a small pulmonary artery of the dog, that contrast material appeared in a pulmonary vein having bypassed the capillary bed. This bypass could be seen to continue after the catheter had been partially withdrawn.
}

be expected to occur with progressive increases in the cardiac output (24) to a previously established critical limit $(1,5)$, it is an unexpected finding in those situations where the exercise outputs remained steady. Some or all of the mechanisms discussed above would need to be invoked. It is also of interest that the estimated total systemic resistance, on the other hand, appears to be predominantly related to the level of the cardiac output. This may be taken to indicate that specific adaptive mechanisms are operative in the cardiopulmonary bed other than would be reflected in a determination of total body vascular resistance, or that baroreceptors in the systemic circulation do not appear to bear a measurable influence on the pulmonary bed.

It is apparent that in using the term "steady state" the exact moieties to which reference is made must be defined. Thus while one may speak of "steady state" of total body oxygen consumption and cardiac output during exercise, the same concept is not applicable to pulmonary artery pressure and total pulmonary resistance within the time limits and work loads defined in these experiments.

Finally, the minute-to-minute pressure levels recorded in these studies reconcile the divergent opinions regarding the changes in pulmonary artery pressure induced by a degree of exercise insufficient to increase the pulmonary blood flow more than two or three-fold $(1,5)$ in normal man. The data will vary according to variability in patients and in methods used-the time of pressure sampling, the number of pressure points recorded, and, to some critical limit, the amount and duration of the imposed work load. It is suggested that just as the validity of cardiac output by the Fick method is insured by matching duplicate determinations, the accuracy of reported pressure changes should be increased by the recording of multiple points for any recorded value and a definition of the sampling time.

\section{SUMMARY AND CONCLUSIONS}

A 39-minute period of mild exercise (850 footpounds per minute) resulted in the maintenance of steady state, as gauged by the oxygen consumption and cardiac output, for about 23 minutes in 7 and for at least 13 minutes in 9 of 10 normal 
subjects. In the tenth subject it was not possible to achieve a steady state of exercise response. In 2 additional subjects steady state was deliberately altered by doubling the work load after the 29th minute of exercise.

Pulmonary artery pressure rose rapidly to a small degree with the beginning of exercise, but generally began to decline after the sixth or seventh minute. The decline was progressive to the end of the allotted time for exercise, and at times pressures fell below the control resting levels. In the 2 subjects in whom the work load was doubled the decrease was temporarily interrupted by a second rise, but thereafter a pressure decline occurred once again.

Total pulmonary resistance decreased progressively throughout the entire exercise period. The decrease in resistance occurred both in those situations where the cardiac output remained unchanged after the establishment of steady exercise state, and in those in whom the cardiac output increased beyond the first established steady level.

Within the time and work load limits of the reported experiments it is suggested that whereas a reasonable measure of exercise steady state may be maintained relevant to the total body oxygen consumption and the cardiac output, the same cannot be said for the pulmonary artery pressure and the total pulmonary resistance.

\section{REFERENCES}

1. Cournand, A., Riley, R. L., Himmelstein, A., and Austrian, R., Pulmonary circulation and alveolar ventilation-perfusion relationships after pneumonectomy. J. Thoracic Surg., 1950, 19, 80.

2. Dexter, L., Whittenberger, J. L., Haynes, F. W., Goodale, W. T., Gorlin, R., and Sawyer, C. G., Effect of exercise on circulatory dynamics of normal individuals. J. Applied Physiol., 1951, 3, 439.

3. Hickam, J. B., and Cargill, W. H., Effect of exercise on cardiac output and pulmonary arterial pressure in normal persons and in patients with cardiovascular disease and pulmonary emphysema. $\mathrm{J}$. Clin. Invest., 1948, 27, 10.

4. McClement, J. H., Renzetti, A. D., Jr., Carroll, D., Himmelstein, A., and Cournand, A., Cardiopulmonary function in hematogenous pulmonary tuberculosis in patients receiving streptomycin therapy. Am. Rev. Tuberc., 1951, 64, 583.

5. Riley, R. L., Himmelstein, A., Motley, H. L., Weiner, H. M., and Cournand, A., Studies of the pulmonary circulation at rest and during exercise in normal individuals and in patients with chronic pulmonary disease. Am. J. Physiol., 1948, 152, 372.

6. Slonim, N. B., Ravin, A., Balchum, O. J., and Dressler, S. H., The effect of mild exercise in the supine position on the pulmonary arterial pressure of five normal human subjects. J. Clin. Invest., 1954, 33, 1022.

7. Sancetta, S. M., and Kleinerman, J., Effect of mild steady state exercise on total pulmonary resistance of normal subjects and those with isolated aortic valvular lesions. Am. Heart J., 1957, 53, 404.

8. Fishman, A. P., McClement, J., Himmelstein, A., and Cournand, A., Effects of acute anoxia on the circulation and respiration in patients with chronic pulmonary disease studied during the "steady state." J. Clin. Invest., 1952, 31, 770.

9. Cournand, A., Some aspects of the pulmonary circulation in normal man and in chronic cardiopulmonary diseases. Circulation, 1950, 2, 641.

10. Sancetta, S. M., and Carleton, R. J., Jr., Variable resistance bicycle ergometer for performance of recumbent exercise. Rev. Scient. Instruments, 1957, 28, 125.

11. Hickam, J. B., and Frayser, R., Spectrophotometric determination of blood oxygen. J. Biol. Chem., 1949, 180, 457.

12. Sancetta, S. M., Acute hemodynamic effects of total head-down body tilt and hexamethonium in normal and pulmonary emphysematous subjects. J. Lab. \& Clin. Med., 1957, 49, 684.

13. Johnson, S. R., The effect of some anaesthetic agents on the circulation in man, with special reference to the significance of pulmonary blood volume for the circulatory regulation. Acta chir. Scandinav., 1951, Supp. 158.

14. Kleinerman, J., and Sancetta, S. M., Effect of mild steady state exercise on cerebral and general hemodynamics of normal untrained subjects. J. Clin. Invest., 1955, 34, 945.

15. Verzár, F., The regulation of the lung volume and its disturbances. Schweiz. med Wchnschr., 1946, 76, 932.

16. Lilienthal, J. L., Jr., and Riley, R. L., Diseases of the respiratory system; circulation through the lung and diffusion of gases in Annual Review of Medicine, Stanford, Calif., 1954, p. 237.

17. Gordon, D. B., Flasher, J., and Drury, D. R., Size of the largest arterio-venous vessels in various organs. Am. J. Physiol., 1953, 173, 275.

18. Prinzmetal, M., Ornitz, E. M., Jr., Simkin, B., and Berman, H. C., Arterio-venous anastomoses in liver, spleen, and lungs. Am. J. Physiol., 1948, 152, 48.

19. Rahn, H., Stroud, R. C., and Tobin, C. E., Visualization of arterio-venous shunts by cinefluorography in the lungs of normal dogs. Proc. Soc. Exper. Biol. \& Med., 1952, 80, 239. 
20. Haddy, F. J., and Campbell, G. S., Pulmonary vascular resistance in anesthetized dogs. Am. J. Physiol., 1953, 172, 747.

21. Cudkowicz, L., and Armstrong, J. B., The bronchial arteries in pulmonary emphysema. Thorax, 1953, 8, 46.

22. Marchand, P., Gilroy, J. C., and Wilson, V. H., An anatomical study of the bronchial vascular system and its variations in disease. Thorax, 1950, 5, 207.

23. Bradford, J. R., and Dean, H. P., The pulmonary circulation. J. Physiol., 1894, 16, 34.

24. Edwards, W. S., The effects of lung inflation and epinephrine on pulmonary vascular resistance. Am. J. Physiol., 1951, 167, 756. 